\title{
Demo: A Reconfigurable 2-D Wireless Charging System
}

Kazunobu Sumiya

The University of Tokyo, Japan

sumiya@akg.t.u-tokyo.ac.jp

Takuya Sasatan

The University of Tokyo, Japan

JSPS Research Fellow

sasatani@akg.t.u-tokyo.ac.jp

\section{Yuki Nishizawa}

The University of Tokyo, Japan

nishizawa@akg.t.u-tokyo.ac.jp
Kenji Tsushio

The University of Tokyo, Japan

tsushio@akg.t.u-tokyo.ac.jp

Yoshiaki Narusue

The University of Tokyo, Japan narusue@mlab.t.u-tokyo.ac.jp

\section{Yoshihiro Kawahara}

The University of Tokyo, Japan kawahara@akg.t.u-tokyo.ac.jp

Permission to make digital or hard copies of part or all of this work for personal or classroom use is granted without fee provided that copies are not made or distributed for profit or commercial advantage and that copies bear this notice and the full citation on the first page. Copyrights for third-party components of this work must be honored. For all other uses, contact the owner/author(s).

Copyright held by the owner/author(s)

UbiComp/ISWC'18 Adjunct, October 8-12, 2018, Singapore, Singapore ACM 978-1-4503-5966-5/18/10.

https://doi.org/10.1145/3267305.3267632

\begin{abstract}
Wireless charging pads such as Qi are rapidly gaining ground, but its limited power supply range still requires precise placement on a specific point. 2-D wireless power transfer (WPT) sheets consisting of coil arrays are one well-known solution for this issue [3]. However, these approaches require custom-made designs by experts; what we need is a system that can be reconfigured by simply placing ready-made modules on the intended surface.

In this demo, we present "Alvus", a reconfigurable 2-D wireless charging system which enables such simple construction of wireless charging surfaces. Alvus is based on multihop WPT, which constructs "virtual power codes" and consists of three types of ready-made resonator modules: transmitter, relay, and receiver. Our system instantly and interactively forms wireless charging surface on everyday objects (e.g. floor, wall, table, etc.) only by placing the resonator modules.
\end{abstract}

\section{Author Keywords}

Ubiquitous Wiress Charging; Multiple Target Devices; Reconstructable Charging Area; Dynamic Power Routing.

\section{ACM Classification Keywords}

B.0 [Hardware]: General 


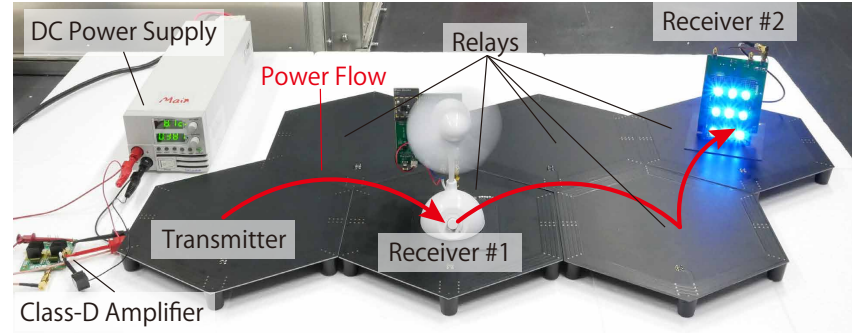

Figure 1: The overview of the reconfigurable 2-D wireless charging system.

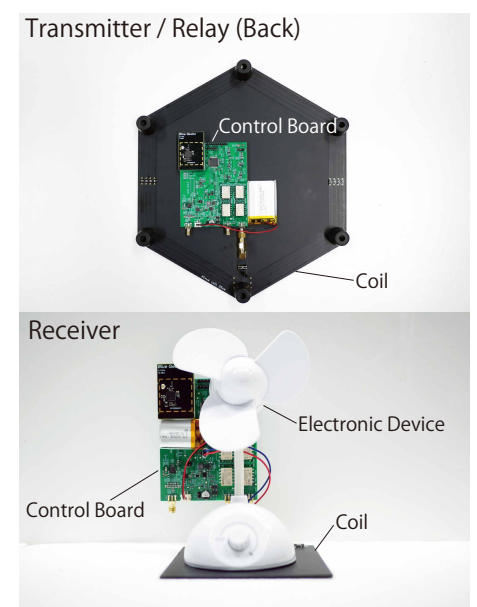

Figure 2: Implemented resonator modules.

\section{System Overview}

The overview of Alvus is shown in Fig. 1. Alvus utilizes the phenomenon called "multihop" to extend the range of WPT [2]; the power "hops" between relay resonators to produce a "virtual" energy route as shown in Fig. 1.

Key Concept and The Primitive Building Blocks of Alvus The key idea of Alvus is to construct a surface packed with programmable (i.e., can enable/disable the relay effect) $L C$ resonator modules and dynamically configure the route of power delivery through enabling/disabling the relay effect of each module. Since this power route is virtual and reprogrammable, $\mathrm{RX}$ (receiver) placed anywhere on the 2$D$ surface can receive the power. Based on this idea, we provide three types of resonator modules, which work as building blocks for Alvus:

1. Transmitter module: The only module connected to the power source. When an RF current oscillating at the resonant frequency $f_{0}=6.78 \mathrm{MHz}$ is fed into this module, a magnetic field is generated and supplies power to adjacent relay resonator modules.

2. Relay module: The module which relays power from the previous module to the next module. The re- (a)

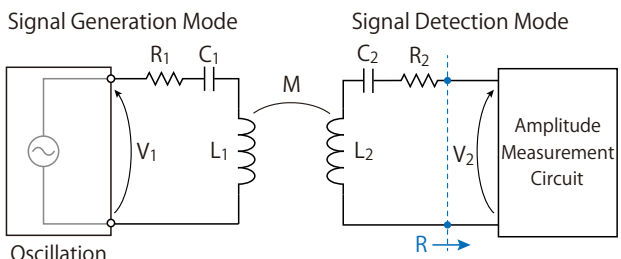

$$
\begin{gathered}
\text { Oscillation } \\
\text { Circuit }
\end{gathered}
$$

(b)

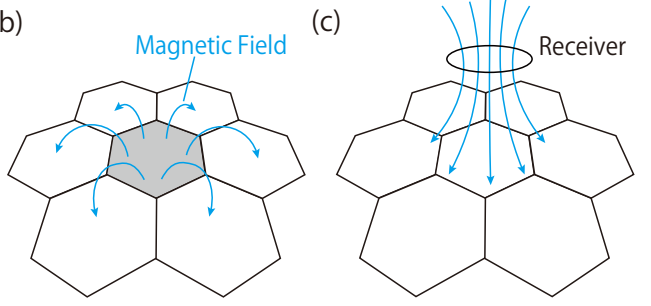

Figure 3: Resonator Detection. (a): Equivalent circuit. (b) Relay detection. (c) Receiver localization.

lay effect can be enabled/disabled by switching the $L C$ resonator between short (enable)/open (disable) state.

3. Receiver module: The module which receives the power from the nearest relay module. The received power is rectified and converted to DC; this DC is supplied to the load device.

Each module has a wireless communication module and is controlled by a central control unit via the wireless communication. We assume a setup that the transmitter module equipped together with the central control unit is placed near the power outlet and the relay modules are placed around the transmitter module to freely extend the WPT enabled area to form an intended shape. 

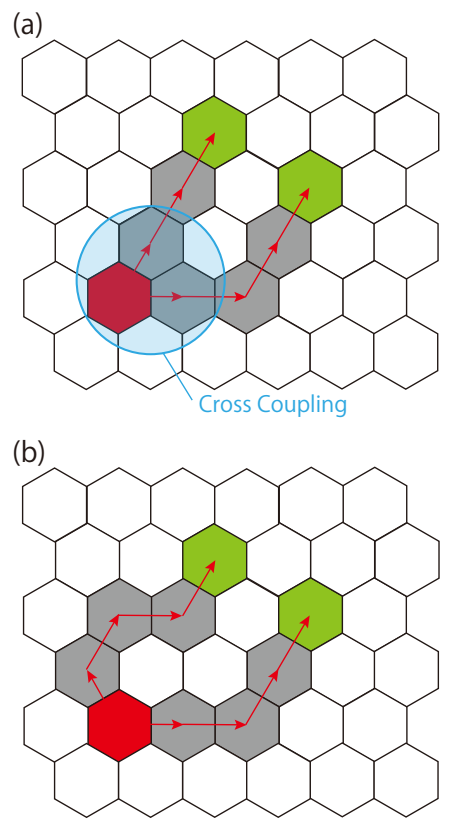

Figure 4: Examples of the power-supply route from the transmitter (red) to two receivers (green). (a) Improper routing. The cross-coupling (shown in the blue circle) degrades power transmission efficiency. (b) Proper routing generated by Alvus.
Design Objectives and Operation Procedure

To construct a reconfigurable 2-D WPT system based on multihop WPT, we set the design objectives of Alvus as the automatic execution of the following functions:

1. Module Map Generation: Detect the placement of the modules, which constructs a 2-D surface.

2. Receiver Localization: Detect the position of the RX when it is on the constructed WPT surface.

3. Dynamic Power Routing: Dynamically configure the state of the modules and construct power routes to efficiently deliver power from an RF power source to the $\mathrm{RX}$

4. Powering Each Module: Deliver the power required for operation on each unit from the RF power source wirelessly.

The basic mechanisms used for these functions are described below.

\section{Module Map Generation}

Detecting the position of relay modules and generating the map is the first function operated in the initialization process. First, the central control unit commands a single module (module in the signal generation mode [See Fig. 3(a)]) to generate a magnetic field oscillating at the resonant frequency $f_{0}=\omega_{0} / 2 \pi$, while the remaining modules (modules in the signal detection mode) are instructed to measure the induced voltage [See Fig. 3(b)]. Assuming that the impedance of the module in the signal detection mode is high enough and non-ideal effects such as cross-coupling can be neglected, the voltage induced to the module in the signal detection mode $\left|V_{2}\right|$ shown in Fig. 3(a) can be approximated as follows [1]

$$
\left|V_{2}\right| \sim \frac{\omega_{0} M}{R_{1}}\left|V_{1}\right|
$$

Here, $M$ is a mutual inductance between the modules in the signal generation mode and the signal detection mode; this is the parameter we intend to extract in this procedure. $V_{1}$ is the voltage applied to the module in the signal generation mode. Since $\omega_{0}, R_{1},\left|V_{1}\right|$ are all known parameters, $M$ can be obtained using the measured value of $\left|V_{2}\right| ; M$ has positive correlation with coupling between the modules, therefore the "closeness in the terms of electromagnetics" can be determined by this value. By repetitively performing this procedure so that every module plays the role of the signal generation mode, a full map of modules, which is a non-directed graph with the coupling used as weight can be obtained.

\section{Receiver Localization}

The voltage supplied to the receiver is continuously monitored when supplying power; if this voltage falls below the pre-defined threshold value, the receiver sends a request for receiver localization to the central control unit. This voltage drop happens in situations such as when (i) the receiver module is placed on the WPT surface for the first time and the route needs to be constructed or when (ii) the receiver module is moved and the route needs to be changed.

The receiver localization procedure uses the same mechanism as map generation. When the receiver localization is requested, the central control unit commands the receiver module to work in the signal generation mode, while the transmitter and relay modules work in the signal detection mode [See Fig. 3(c)]. Through the measured voltage values, the module which most strongly couples with the receiver is determined.

Dynamic Power Routing

Based on the map of transmitter/relay modules and the location of the receiver module, the central control unit generates a route for multihop power delivery and configure the 


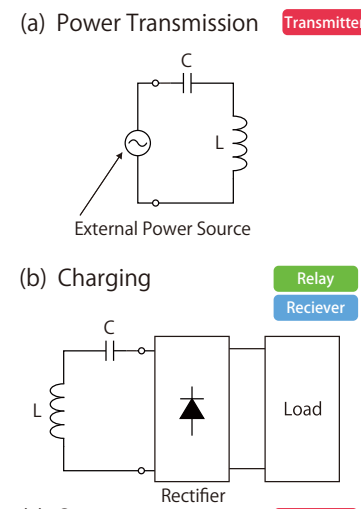

(c) Open

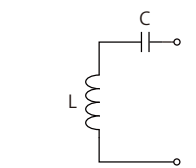

(d) Short

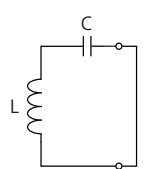

(e) Signal Generation

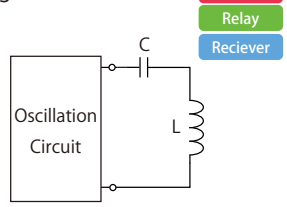

(f) Signal Detection

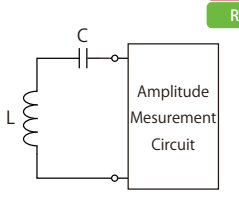

Figure 5: Required functions of Alvus resonator modules. relay modules to establish the route.

Alvus determines a route to transmit power that avoids the degradation of transmission efficiency as much as possible, by considering the placement of resonators [see Fig. 4]

Unexpected error factors such as large metallic objects placed on the route can degrade the system performance or even cause safety issues. Therefore, a validation procedure runs to check if the generated route is still valid before power delivery. This validation process simply runs the module map generation process for only the modules in the route; if the obtained value is not consistent with the values saved in the map, the module is considered out of order, the module is removed from the map, and the route is regenerated.

\section{Required Functions of Modules}

Now that the work flow is defined and the mechanism for each process is considered, the next thing to discuss is the primitive functions that the modules should be capable of. The six functions listed below and Fig. 5 are the functions necessary to enable the aforementioned operation procedure; which is implemented for demonstration.

(a) Power Transmission: Control the ON/OFF of the RF power input. (Required in: Transmitter module.)

(b) Charging: Receive power via the ambient magnetic field, rectify, and supply power to the battery and the load. (Required in: Relay and receiver module.)

(c) Open: Make the impedance of the resonator high and disable the multihop effect. This minimizes interference with nearby modules. (Required in: All modules.) (d) Short: Make the impedance of the resonator low and enable the multihop effect. (Required in: Relay module.)

(e) Signal Generation: Generate the magnetic field for map generation and receiver localization (Required in: All modules.)

(f) Signal Detection: Detect the magnetic field for map generation and receiver localization (Required in: Transmitter and receiver module.)

\section{Acknowledgements}

This work was supported by JST ERATO Grant Number JPMJER1501 and Grant-in-Aid for JSPS Fellows JP18J22537.

\section{REFERENCES}

1. Arata Hashizume, Yoshiaki Narusue, Yoshihiro Kawahara, and Tohru Asami. 2017. Receiver localization for a wireless power transfer system with a $2 D$ relay resonator array. In 2017 IEEE International Conference on Computational Electromagnetics (ICCEM). 127-129.

2. Yoshiaki Narusue, Yoshihiro Kawahara, and Tohru Asami. 2013. Impedance matching method for any-hop straight wireless power transmission using magnetic resonance. In 2013 IEEE Radio and Wireless Symposium. 193-195.

3. Tsuyoshi Sekitani, Makoto Takamiya, Yoshiaki Noguchi, Shintaro Nakano, Yusaku Kato, Takayasu Sakurai, and Takao Someya. 2007. A large-area wireless power-transmission sheet using printed organic transistors and plastic MEMS switches. Nature materials 6, 6 (2007), 413 . 